\title{
Editorial: RMP: Looking Forward
}

Ninety years! As the first spinoff of the Physical Review launched in 1929, Reviews of Modern Physics (RMP) has tracked the progress of physics during a time of explosive growth of the field. Originally one journal, the Physical Review has not only split into the more specialized journals we know today, but the scope, breadth, and depth of topics covered could hardly have been imagined by John Tate, Sr., the editor in chief who started RMP. Yet RMP is still published quarterly. So why hasn't RMP grown at the same prodigious rate? The research that informs these reviews is progressing faster, but writing a review that is broadly accessible and that will remain relevant for decades has therefore become more difficult. RMP's role is to find authors who are up to this challenge.

At the April 2019 editorial board meeting we resolved to reaffirm our mission of providing the community with articles that capture the essence of the field, with introductions and conclusions that are accessible to students (our most junior colleagues who will carry science into the future) and that communicate how and why the topic has fascinated scientists and provided an arena for new paradigms, theories, and connections between subfields. To this end, we will implement even higher standards for our submissions: not only will submitted manuscripts be reviewed by experts, they will also be reviewed by qualified non-experts from nearby subfields to ensure broad accessibility. Though this is, of course, a greater burden on our referees and authors, we believe that it is necessary to publish "classic" articles and keep RMP vital for many years to come.

We look forward to continuing to serve the community.

Randall D. Kamien
Hiroaki Aihara
Dietrich Belitz
Debbie Brodbar
A. H Castro Neto
Margaret S. Cheung
William D. Collins
Marjolein Dijkstra
David DiVincenzo
Paul D. Grannis
Arthur F. Hebard
Vicky Kalogera
Igor Klebanov
Wim Leemans
Klaus Mølmer
Witold Nazarewicz
Pierre Ramond
Roxanne Springer
Anthony F. Starace
Friedel Thielemann

Published 1 July 2019

DOI: 10.1103/RevModPhys.91.030001 